\title{
Pleural fluid Adenosine deaminase activity - Can it be a diagnostic biomarker?
}

\author{
VinayBharat ${ }^{\mathrm{a}}$, B. K. Gupta ${ }^{\mathrm{b}}$, ParulGoel ${ }^{\mathrm{c}}$, Kahkasha ${ }^{\mathrm{d}}$, PreetiShukla ${ }^{\mathrm{e}}$, \\ ${ }^{a}$ M.B;B.S; D.C.P.; M. D, Professor, Department of Pathology, Subharti Medical College, Swami \\ VivakanandSubharti University, Meerut-250005, India \\ ${ }^{b}$ M.B;B.S; D.T.C.D.; Ph D, Professor, Department of Biochemistry, Subharti Medical College, Swami \\ VivakanandSubharti University, Meerut- 250005, India

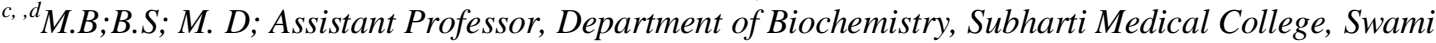 \\ VivakanandSubharti University, Meerut- 250005, India \\ ${ }^{e}$ Research Scholar, Department of Biochemistry, Subharti Medical College, Swami VivakanandSubharti \\ University, Meerut- 250005, India
}

\begin{abstract}
Aim: To evaluate pleural fluid Adenosine deaminase as the diagnostic marker for tubercular pleural disease.

Patients and Methods:New patients ( $n=160)$ with pleural effusion were divided into tubercular $(n=92)$ and non-tubercular ( $n=68)$ groups. Non-tubercular group was further divided into patients having exudative effusion (n=46) and patients having transudative effusion $(n=22)$. Patients with exudative effusion included those with adenocarcinoma $(n=21)$, non-tuberculous empyema $(n=11)$, parapneumonic effusion: $(n=9)$ and lymphomas ( $n=5)$ while patients having transudative effusion included those with congestive heart failure $(n=13)$, cirrhosis of liver $(n=6)$ and with nephrotic syndrome $(n=3)$. Pleural fluid was aspirated from all patients and tested for $A D A$.

Results: In tubercular group Mean $\pm S D$ of ADA value was $67.78 \pm 37.39$ and it ranged between $8.8-260.0$; in non-tubercular group collectively it was $22.17 \pm 15.11$ and ranged between $6.0-102.0$. The performance of ADA for diagnosis of tuberculous pleural effusions in regards to the $95 \%$ confidence intervals and cut-off levels above $40.0 \mathrm{IU} / \mathrm{L}$ resulted in $88.04 \%$ sensitivity, $91.12 \%$ specificity, $95.25 \%$ positive predictive value, $85.33 \%$ negative predictive value, $90.63 \%$ diagnostic accuracy, 14.7 positive likelihood ratio, 0.13 negative likelihood ratio and 11.78 diagnostic odds ratio. The prevalence of disease in the studied population was $57.5 \%$.

Conclusion: It is concluded that ADA estimation in pleural fluid is not only simple, inexpensive and rapid but also fairly specific and sensitive method for diagnosing tuberculous etiology in patients of pleural disease. ADA activity in the pleural fluid can be a diagnostic biomarker and for this reason ADA estimation in pleural fluid may find a place as a routine investigation.
\end{abstract}

Keywords:Tuberculosis, Pleural effusion, Adenosine deaminase, Diagnostic, Biomarker,

\section{Introduction:}

Tuberculosis is one of the commonest chronic infectious diseases, which is highly endemic killing approximately five lakh patients every year in India [1]. It usually affects lungs but cases of extrapulmonary tuberculosis are not rare. Delay in diagnosis and in initiating treatment results in poor prognosis and sequelae in upto $25 \%$ of cases [2].

Pulmonary Tuberculosis (TB) can be confirmed by serial sputum examination and diagnosed easily, but diagnosing extra-Pulmonary TB often becomes difficult since the specificity and sensitivity of available non-invasive methods is low. Pleural tuberculosis is an extrapulmonary form of tuberculosis affecting $15 \%$ of the total number of cases. It is the second most frequent clinical presentation of human tuberculosis $[1,2]$.

Pleural effusion is a common problem in clinics and can result due to a number of diseases. The available tests and procedures for the confirmation of its etiology are ineffective in majority of cases. Thus, there is a need for a sensitive and specific test that is reliable and rapid [3].

Tubercular pleural effusion is usually a result of delayed type hypersensitivity to proteins of the bacterium, but owing to low bacterial load in the pleural space, the aspirated fluids when analyzed for direct evidence of disease show very low acid fast bacilli (AFB) positivity rate. Direct examination of pleural fluid by Zeihl-Neelsen staining requires bacillary densities of $10,000 / \mathrm{mL}$ [3] and, therefore, detects AFB in less than $10 \%$ of cases. AFB culture is expensive, requires long incubation period and is positive in less than $25 \%$ cases. ELISA, PCR, TB IgG / IgM and interferon are very expensive tests and beyond the reach of the masses. Pleural biopsy although considered as the gold standard for diagnosis of pleural disease, is still not a routine medical procedure due to its invasive nature. 
Adenosine and deoxyadenosineis converted into inosine and dexyinosine in the purine salvage pathway with the release of ammonia; Adenosine deaminase (ADA) is an enzyme that catalyzes this conversion. It plays important role in differentiating lymphoid cells and is present in abundance in active T-lymphocytes where its concentration is inversely proportional to the degree of differentiation [4]. The enzyme activity increases during mitogenic and antigenic responses of lymphocytes, and some earlier reports have even shown that T-lymphocyte blastogenesis can be inhibited by inhibitors of ADA. Likewise, deficiency of adenosine deaminase is associated with severe defects in the cell-mediated and humoral immunity, predisposing the patient to opportunistic infections. Adenosine deaminase has been proposed as an important marker for pleural TB as its activity correlates with CD4+ $\mathrm{T}$ cell infiltration in the pleura and pleural fluid. ADA is now being recognized as a marker of cell-mediated immunity particularly as a marker of T-lymphocyte activation [4, 5]. Elevated levels of ADA in tuberculous effusions have been noted by several authors and because it is an easy little-invasive investigation, it is frequently considered as a diagnostic aid in such cases. ADA levels have also been considered by several researchers to differentiate tubercular disease from non-tubercular effusions with a sensitivity of $90-$ $100 \%$ and specificity $89-100 \%$ [5-7].

We planned the present study to further investigate the role of pleural fluid ADA level in confirmation of the diagnosis of TB and to evaluate; if it can be a diagnostic biomarker.

\section{Patients and Methods:}

Patients with pleural effusion; attending outdoor/indoor of Respiratory disease of ChatrapatiShivajiSubhartiHospital, Meerut, India between August 2010 to November 2012 were enrolled for the present study. The study was approved by the Ethics Committee of the Institute. Written informed consent was taken from all patients participating in the study.

Fresh pleural effusion patients, with no previous history of medication were included in the study. Detailed present, past, drug and family history was taken, a thorough physical examination, routine and special investigations and radiographic evaluation was done for each patient. Closed pleural biopsy was taken with the help of a Cope's needle and atleast three samples were taken from the same region of thorax. Broncho-alveolar lavage (BAL) was done through a bronchoscope in specific patients.

Thoracocentesis was done under aseptic conditions as per standard protocol for all of them and pleural fluid so aspirated was collected in a sterile tube without an anticoagulant for total ADA quantification, routine analysis and cytology.

\section{Patients were classified into various groups as per the following criteria [8]:}

1. Presence of first or more than one of the following criteria was adopted to include the patient into tubercular group;

A. Bacteriological proof of the presence of Mycobacterium tuberculosis through direct smear / culture of pleural fluid/sputum/BAL.

B. Pleural biopsy showing caseating granulomas.

C. Clinico-Radiological findings consistent with TB.

D. Definite clinical and radiological improvement in one month after specific anti-tubercular treatment.

E. History of contact with current disease and positive reaction ( $>10 \mathrm{~mm}$ induration) to 5 tuberculin unit (TU) purified protein derivative (PPD).

2. In case patient did not fulfill the above criteria, they were non-tubercular and were further divided into different subgroups depending upon the following diagnostic criteria;

1) Adenocarcinoma, if their pleural fluid/BAL cytology showed presence of few malignant cells with a tendency to form smoothly contoured cohesive groups composed of large cells with eccentric, malignant appearing nuclei, prominent nucleoli and vacuolated cytoplasm [9].

2) Empyema, if the pleural fluid was pus (thick, opaque, yellowish-white, viscous fluid) loculated or otherwise, was purulent and/or had a positive Gram stain or culture. Pleural infection was indicated by pleural acidosis associated with raised lactate dehydrogenase (LDH) and low glucose levels [10].

3) Parapneumonic, if their pleural fluid was secondary to pneumonia, lung abscess or bronchiectasis in patients with cough, fever, and in cases where radiographic pulmonary infiltrate disappeared with antibiotic treatment [10].

4) Lymphoma, if their pleural fluid/BAL cytology showed presence of discrete monomorphic malignant cells with massive necrosis, pyknosis and fragmentation of nuclei [11].

5) $\mathrm{CHF}$, if the patient had enlarged cardiac shadow on plain X-ray chest with clinical or echocardiographic evidence of cardiac dysfunction, with one or more of the following alterations: pulmonary venous congestion on radiography, peripheral edema, tachycardia or ventricular gallop [12].

6) Cirrhosis liver, if the patient had ascites, splenomegaly, oesophagealvarices, portal hypertension, moderate increase in serum bilirubin and SGPT and increased prothrombin time [13]. 
7) Nephrotic syndrome, if the patient had generalized anasarca, proteinuria more than $3.5 \mathrm{gm} / \mathrm{day}$, hypoalbuminemia with altered $\mathrm{A} / \mathrm{G}$ ratio and hyperlipidemia [14].

Pleural fluid (PF) was classified as [15]:

a) Exudative if $\mathrm{PF} /$ serum protein $>0.5 ; \mathrm{PF} /$ serum $\mathrm{LDH}>0.6$ and $\mathrm{PF} \mathrm{LDH}>2 / 3$ upper normal limit for serum.

b) Transudative if $\mathrm{PF} /$ serum protein $<0.5 ; \mathrm{PF} /$ serum $\mathrm{LDH}<0.6$ and $\mathrm{PF} \mathrm{LDH}<2 / 3$ upper normal limit for serum.

\section{Exclusion criteria:}

The patients who presented with any of the following criteria were not included in the study:

1) Absolute contraindication or refusal to undergo thoracocentesis.

2) With recurrent effusion.

3) History of previous medication for $\mathrm{TB}$, adenocarcinoma, empyema, parapneunomia, lymphoma, CHF, Cirrhosis liver or nephrotic syndrome.

4) On corticosteroid medication.

5) With hemolysis in pleural fluid.

6) With renal failure, HIV infection or any immunodeficiency disease.

7) No diagnosis could be established.

\section{Adenosine deaminase (ADA) assay:}

ADA in pleural fluid was assayed by the method of Giusti [16] with slight modifications. The technique is a colorimetric method based on the measurement of ammonia by Berthelot reaction, which is produced when ADA reacts with excess adenosine. The formation of blue indophenol was measured at $628 \mathrm{~nm}$ with a spectrophotometer. All solutions for the assay were prepared in house, using triple distilled water and were standardized before subjecting them for ADA estimation. Ammonium sulphate $(75 \mu \mathrm{M})$ was used as ammonium standard. One IU of total ADA was defined as the amount of enzyme required to release $1 \mu \mathrm{mol}$ ammonia per minute from adenosine under standard assay conditions. The enzyme is stable for at least 24 hours at $25^{\circ} \mathrm{C}$, for 7 days at $4^{\circ} \mathrm{C}$, and for 3 months at $-20^{\circ} \mathrm{C}[16,17]$. All the samples were stored in a deep freezer at $20^{\circ} \mathrm{C}$ till analyzed. A positive control sample and two negative control samples for which the ADA value was known were included in each group of pleural fluid sample analyzed. The optimal cut off value of ADA was determined using the receiver operating characteristic (ROC) curve as $>40 \mathrm{IU} / \mathrm{L}$.

\section{Statistical Analysis [18, 19]:}

All data are expressed as mean and standard deviations. ADA levels were measured in patients with pleural effusions due to TB and also in patients with pleural effusions of non-tuberculous origin as the controls. To compare the differences in ADA levels between the two groups, we performed the Z-test (Double-sample mean) to test the significance at $1 \%$ level of significance. Further, Pearson chi-square test without a Yates correction was also applied to find the association between the tubercular and non-tubercular groups at $1 \%$ level of significance. Also, Kruskall-Wallis test was used to compare tuberculosis versus nontuberculous groups. A two- tailed p-value less than 0.01 was considered statistically significant. On the basis of the ADA results obtained from pleural TB and control groups, the sensitivity, specificity, accuracy, predictive values, likelihood ratio and diagnostic odds ratio of the test were calculated in order to establish the potential utility of ADA as the diagnostic marker for patients with pleural TB.

\section{Results:}

Patients ( $\mathrm{n}=160)$ were divided into two groups i.e., tubercular $(\mathrm{n}=92)$ and non-tubercular $(\mathrm{n}=68)$ on the basis of their diagnosis. Non-tubercular group was further divided into two groups i.e., patients having exudative effusion $(n=46)$ and patients having transudative effusion $(n=22)$. Patients with exudative effusion included those with adenocarcinoma $(\mathrm{n}=21)$, non-tuberculous empyema $(\mathrm{n}=11)$, parapneumonic effusion: both simple and complicated $(n=9)$ and lymphomas $(n=5)$ while patients having transudative effusion included those with congestive heart failure (CHF) $(n=13)$, cirrhosis of liver $(n=6)$ and with nephrotic syndrome $(n=3)$ (table 1).

In tubercular group Mean \pm SD of ADA value was $67.78 \pm 37.39$ and it ranged between $8.8-260.0$; in non-tubercular group Mean \pm SD of ADA was $22.17 \pm 15.11$ and it ranged between $6.0-102.0$ collectively; Mean \pm SD of ADA for exudative effusion was $25.14 \pm 16.99$ and it ranged between $7.5-102.0$ while in patients with transudative effusion it was $18.60 \pm 7.86$ and ranged between $6.0-34.0$ (Table 1).

In adenocarcinoma patients Mean \pm SD of ADA value was $22.90 \pm 9.06$ and it ranged between $8.8-$ 42.0; in non-tubercular empyema patients it was $36.83 \pm 28.89$ and ranged between $10.0-102.00$; for parapneumonic patients it was $17.87 \pm 9.20$ ranging between $7.5-32.4$ while in lymphoma patients Mean \pm SD of ADA value was $21.92 \pm 7.00$ with ADA values ranging between $16.0-34.0$ (Table 1). 
In CHF patients Mean \pm SD of ADA value was $19.87 \pm 6.70$ and it ranged between $11.8-30.2$; in Cirrhosis patients it was $21.36 \pm 8.08$ and ranged between $12.0-34.0$; while in nephrotic syndrome patients Mean $\pm \mathrm{SD}$ of ADA value was $7.60 \pm 1.44$ and it ranged between $6.0-8.8$ (Table 1).

The application of Z-test (double-sample mean) revealed a significant difference between tubercular and non-tubercular groups as well as with tubercular and different subgroups of non-tubercular group at $1 \%$ level of significance i.e., (P<.01). (Table 1; column 5).

Further, chi-square test statistic also showed a significant difference between tubercular and nontubercular groups at $1 \%$ level of significance i.e., $(\mathrm{P}<0.1)($ chi-square $=93.52, \mathrm{p}<0.01)$. Moreover, KruskalWallis test also showed a significant difference between tubercular and different subgroups of non-tubercular groups at $1 \%$ level of significance i.e., $(\mathrm{P}<.01)(\mathrm{H}=113.71, \mathrm{p}<0.01)$. (Table 1$)$.

The performance of ADA for diagnosis of tuberculous pleural effusions in regards to the $95 \%$ confidence intervals and cut-off levels above 40.0 IU/L resulted in $88.04 \%$ sensitivity, $91.12 \%$ specificity, $95.25 \%$ positive predictive value, $85.33 \%$ negative predictive value, $90.63 \%$ diagnostic accuracy, 14.7 positive likelihood ratio, 0.13 negative likelihood ratio and 11.78 diagnostic odds ratio, the prevalence of disease in the studied population was $57.5 \%$ (table 2 ).

In our study there were few cases $(n=4)$ in nontubercular group which showed ADA levels more than the cut off value (false-positive cases), they were adenocarcinoma $(\mathrm{n}=1)$ and empyema from bacterial infections $(\mathrm{n}=3)$.

\section{Discussion:}

Pleural effusion is a common problem in clinics and can result due to a number of diseases. Many times in the absence of reliable test reports diagnosis cannot be confirmed and this results in delay in starting proper treatment ultimately leading to progression of the disease and involvement of other organs and more complications. At times patients are treated on clinical impression rather than on laboratory test results which may lead to overtreatment [10].

The conventional diagnosis of pleural tuberculosis, is usually based on an observation of epithelioidgranulomas in pleural tissue biopsy [10]. Acid-fast staining of pleural fluid is a rapid, inexpensive method for diagnosing pleural tuberculosis, but its sensitivity is as low as 0 to $20 \%$. The isolation of Mycobacterium tuberculosis in cultured pleural fluid or tissue biopsy specimens permits a definite diagnosis, but again, the sensitivity is low and results can take as long as 2 to 6 weeks to arrive [10].

New techniques have been reported to facilitate the diagnosis of tuberculous pleuritis; like adenosine deaminase activity, interferon gamma levels and polymerase chain reaction. Surprisingly, using polymerase chain reaction has a relatively low sensitivity in pleural fluid (42\% to $81 \%)$ and is fairly expensive [20, 21]. The sensitivity of an elevated interferon gamma level appears to be better ( $89 \%$ to $99 \%$ ), but there have been relatively few studies evaluating its use and the assay is expensive [21].

Reid et al.[18] pointed out serious methodological limitations in the research evaluating diagnostic tests; published in the most prestigious international scientific clinical journals. To prevent errors in this ADA study we applied the methodological criteria recommended by Reid et al and by the Standards for Reporting of Diagnostic Accuracy-STARD[19]. The standard measures of validity are sensitivity and specificity. Other closely related measures are positive and negative predictive value, diagnostic odds ratio, likelihood ratios and the area under a ROC curve[19].

Our study (table 1) revealed high levels of ADA activity in the pleural fluid of tuberculous patients compared to a control group (chi-square $=93.52, \mathrm{p}<0.01$ ). On the basis of the ADA results obtained for the TB and non-TB groups, the sensitivity, specificity, predictive values, likelihood ratio and diagnostic odds ratios of the test were calculated (Table 2), which established the potential utility of ADA as the diagnostic biomarker for patients with pleural TB.

In our study there were few cases $(n=4)$ in nontubercular group which showed ADA levels more than the cut off value (false-positive cases), they were adenocarcinoma $(n=1)$ and empyema from bacterial infections $(n=3)$. Based on this fact we tried to find out the statistical performance so that the question addressed, "Pleural fluid Adenosine deaminase activity - Can it be a diagnostic biomarker?" be answered.

The performance of ADA for diagnosis of tuberculous pleural effusions as regards to the $95 \%$ confidence intervals and cut-off levels above 40.0 IU/L resulted in $88.04 \%$ sensitivity, $91.12 \%$ specificity, $95.25 \%$ positive predictive value, $85.33 \%$ negative predictive value, $90.63 \%$ diagnostic accuracy, 14.7 positive likelihood ratio, 0.13 negative likelihood ratio and 11.78 diagnostic odds ratio (table 2). The prevalence of disease in the studied population was $57.5 \%$ (table 2).

Several studies have suggested that elevated pleural fluid ADA level predicts tuberculous pleuritis with a sensitivity of $90-100 \%$ and a specificity of $89-100 \%$ when measured by the Giusti method. The reported cut off value for ADA in these studies varies from 35.0 to $70.0 \mathrm{U} / \mathrm{L}[22$ - 27]. 
Bossuyt PM et al and De Oliveira HG et al demonstrated specificity of ADA for the diagnosis of pleural tuberculosis increased to $99.0 \%$ in case false positive are included[19, 25]. The specificity of ADA for pleural TB may be increased in clinical practice when false-positive cases remain undiagnosed by other tests. An example in the present study is 3 cases of empyema and one case of adenocarcinoma who had ADA levels above the cut off mark (false positive); but they were diagnosed by other means like clinical examination, imaging and pleural fluid examination.

Gupta et at[28] observed that ADA levels in non tuberculous disease rarely exceeded the cut-off value; set for tuberculous disease. They[29] have further observed that ADA estimation is not only a fairly sensitive and specific test (more than 90\%), helpful in differentiating tubercular from non-tubercular etiology; both in pulmonary and extra-pulmonary disease but is also simple, inexpensive and rapid. For these reasons this test may help in early diagnosis, improve the prognosis and reduce spread of disease and sequelae.

Although Tuon et at[30] in their meta-analysis concluded that, ADA cannot distinguish between bacterial meningitis and TBM, but their observations are based on studies having heterogeneity, publication bias and nonstandardized method for ADA estimation and so further studies are needed to obtain reliable conclusions.

Gupta et al[8,31] had further found the sensitivity of this test to be $94.73 \%$; specificity $90.47 \%$, positive predictive value is $90.00 \%$ and negative predictive value $95.00 \%$ and they had concluded that ADA estimation in CSF is not only simple, inexpensive and rapid but also fairly specific method for making a diagnosis of tubercular meningitis in children and may be established as a marker for diagnosis of the same.

Our findings are similar to other researchers[28-30, 32]. The high sensitivity and specificity for adenosine deaminase, can contribute to a diagnosis of pleural tuberculosis, and, in many cases, render a pleural biopsy unnecessary. Furthermore a pleural ADA assay is inexpensive, rapid, and simple to perform and is of great value for the diagnosis of tubercular pleuritis and the early initiation of specific treatment.

\section{Conclusion:}

It is concluded that ADA estimation in pleural fluid is not only simple, inexpensive and rapid but also fairly specific and sensitive method for diagnosing tuberculous etiology in patients of pleural disease because of the accuracy parameters demonstrated in this study. ADA activity in the pleural fluid can be a diagnostic biomarker and for this reason ADA estimation in pleural fluid may find a place as a routine investigation.

\section{Acknowledgement:}

We are thankful to Mr. RupeshTiwari, statistician in the department of Preventive Medicine, Subharti Medical College, Meerut, India, for extending technical help in doing all statistical calculations.

Table 1: Distribution of patients; pleural fluid ADA activity and range with Probable values of Z-test; compared with tuberculosis.

\begin{tabular}{|l|l|l|l|l|}
\hline Study Group & Number of Patients & $\begin{array}{l}\text { Mean } \pm \text { SD ADA } \\
(\mathrm{IU} / \mathrm{L})\end{array}$ & $\begin{array}{l}\text { Range of ADA } \\
(\mathrm{IU} / \mathrm{L})\end{array}$ & $\begin{array}{l}\text { Probable values of Z- } \\
\text { test; } \begin{array}{l}\text { compared } \\
\text { with } \\
\text { tuberculosis }\end{array}\end{array}$ \\
\hline Tuberculosis & 92 & $67.78 \pm 37.39$ & $8.8-260.0$ & --------- \\
\hline Non-tuberculosis & 68 & $22.17 \pm 15.11$ & $6.0-102.0$ & $0.0000(\mathrm{P}<.01)^{*}$ \\
\hline Exudative & 46 & $25.14 \pm 16.99$ & $7.5-102.0$ & $0.0000(\mathrm{P}<.01)^{*}$ \\
\hline Adenocarcinomas & 21 & $22.90 \pm 9.06$ & $8.8-42.0$ & $0.0000(\mathrm{P}<.01)^{*}$ \\
\hline $\begin{array}{l}\text { Empyemas non- } \\
\text { tubercular }\end{array}$ & 11 & $36.83 \pm 28.89$ & $10.0-102.00$ & $0.0057(\mathrm{P}<.01)^{*}$ \\
\hline $\begin{array}{l}\text { Parapneumonic simple } \\
\text { and complicated }\end{array}$ & 09 & $17.87 \pm 9.20$ & $7.5-32.4$ & $0.0000(\mathrm{P}<.01)^{*}$ \\
\hline Lymphomas & 05 & $21.92 \pm 7.00$ & $16.0-34.0$ & $0.0000(\mathrm{P}<.01)^{*}$ \\
\hline Transudative & 22 & $18.60 \pm 7.86$ & $6.0-34.0$ & $0.0000(\mathrm{P}<.01)^{*}$ \\
\hline CHF & 13 & $19.87 \pm 6.70$ & $11.8-30.2$ & $0.0000(\mathrm{P}<.01)^{*}$ \\
\hline Cirrhosis & 06 & $21.36 \pm 8.08$ & $12.0-34.0$ & $0.0000(\mathrm{P}<.01)^{*}$ \\
\hline Nephrotic syndrome & 03 & $7.60 \pm 1.44$ & $6.0-8.8$ & $0.0000(\mathrm{P}<.01)^{*}$ \\
\hline
\end{tabular}

$(\mathrm{p}<.01)^{*}$ - shows significant difference at $\alpha=.01$ level of significance.

Table 2: Performance of pleural fluid ADA activity for diagnosis of tuberculous pleural effusions at cut-off levels above $40.0 \mathrm{IU} / \mathrm{L}$.

\begin{tabular}{|l|l|l|}
\hline Statistical indices of diagnostic accuracy & Results & 95\% CI \\
\hline Sensitivity (\%) & 88.04 & $82.61-92.11$ \\
\hline Specificity (\%) & 91.12 & $84.55-96.30$ \\
\hline Positive predictive value (\%) & 95.25 & $89.48-99.38$ \\
\hline
\end{tabular}


Pleural fluid Adenosine deaminase activity - Can it be a diagnostic biomarker?

\begin{tabular}{|l|l|l|}
\hline Negative predictive value (\%) & 85.33 & $80.43-90.17$ \\
\hline Positive likelihood ratio & 14.7 & $13.23-15.80$ \\
\hline Negative likelihood ratio & 0.13 & $0.11-0.31$ \\
\hline Diagnostic odds ratio & 11.78 & $10.98-13.18$ \\
\hline Accuracy (\%) & 90.63 & $85.09-97.12$ \\
\hline Prevalence (\%) & 57.5 & $53.63-63.33$ \\
\hline
\end{tabular}

\section{Reference}

[1]. Garcia-Monco, JC. CNS Tuberculosis. In: NeurologicClinics. Marra, CM (ed.) 1999; 17(4):737-60.

[2]. Mastroianni CM, Paolotti F, Lichtrer MD, Agostino C, Vullo V, Delia S. Cerebrospinal fluid cytokines in patientswith tuberculous meningitis, clinImmunopathology 1997;84:171-176.

[3]. Thwaites G, Chau TTH, Mai NTH, Drobniewski F, McAdam K, Farrar K. Neurologicalaspects of tropical disease: Tuberculous meningitis. J NeurolNeurosurgPsychiatry 2000;68:289-99.

[4]. Tandon, PN. Neurotuberculosis: Clinical aspects. In Neruology in Tripica. Chopra, J.S. and Sawhney, I.M.S. (eds.) Churchill Livingstone Ltd., 358-389.

[5]. Bothamley GH. Serological diagnosis of tuberculosis. Eur. Respir. J 1995;8(Suppl20):676-688.

[6]. Malan C, Donald PR, Golden M and Talard JJF. Adenosine deaminase levels in cerebrospinal fluid in the diagnosis of tuberculous meningitis. J Trop Med Hy1984;87: 33-40.

[7]. Piras MA, Gakis C. Cerebrospinal fluid adenosine deaminase activity in tuberculous meningitis. Enzyme 1973;14:311-17.

[8]. Gupta B K, Bharat A, Bandyopadhyay D, Baruah H. Adenosine deaminase levels in CSF of tuberculous meningitis patients, J Clin Med Res 2010; 2(5):220-24

[9]. Bibbo M. Pleural, Peritoneal and Pericardial Fluids. In Comprehensive Cytopathology, 2nd ed. Philadelphia: W B Saunders Company. p. 590-91.

[10]. Light RW. Clinical Manifestations and Useful Tests. In: Williams \& Wilkins, editor. Pleural Diseases 4th 265ed. Philadelphia: Lippincott; 2001.p. 42-86.

[11]. Bibbo M. Pleural, Peritoneal and Pericardial Fluids. In Comprehensive Cytopathology , 2nd ed. Philadelphia: W B Saunders Company .p. 607.

[12]. Marantz PR, Tobin JN, Smoller SW, Steingart RM, Wexler JP, Budner N et al. The relationship between left ventricular systolic function and congestive heart failure diagnosed by clinical criteria. Circulation, J American Heart Association 1988;77:607-612.

[13]. Mandal AK, Choudhury S. Hepatobiliary System: Jaundice, Hepatitis and other Diseases. In: Text book of Pathology for BDS, 1st ed. New Delhi: Avichal Publishing Company; 2012. p. 467.

[14]. Mandal AK, Choudhury S. Urinary Examination and Renal Diseases. In: Text book of Pathology for BDS, 1st ed. New Delhi: Avichal Publishing Company; 2012. p. 478.

[15]. Light RW. Disorders of the Pleura and Mediastenum. In: Harrison's Principles of Internal Medicine, 17th Edition, McGraw Hill Company; 2008.p. 1658.

[16]. Giusti G. Adenosine deaminase. In: Bergmeyer HU, editor. Methods of Enzymatic Analysis. 2nd ed. New York: Academic Press; 1974. p. 1092-99.

[17]. Neves DD, Silva Junior CT, Preza PCA, Morisson P. Adenosine deaminase activity (ADA) measurement. Pulmao RJ 2004;13(3):182-89.

[18]. Reid MC, Lachs MS, Feinstein AR. Use of methodological standards in diagnostic test research. JAMA 1995;274:645-51.

[19]. Bossuyt PM, Reitsma JB, Bruns DE, Gatsonis CA, Glasziou PP, IrwigLM,et al. Standards for Reporting for Diagnostic Accuracy. The STARD statement for reporting studies of diagnostic accuracy: explanation and elaboration. ClinChem 2003;49:7-18.

[20]. Palomino JC. Nonconventional and new methods in the diagnosis of tuberculosis: feasibility and applicability in the field. EurRespir J 2005;25(2):339-50.

[21]. Villegas MV, Labrada LA, Saravia NG. Evaluation of Polymerase Chain Reaction, Adenosine Deaminase, and Interferon-Gama in Pleural Fluid for the Differential Diagnosis of Pleural Tuberculosis. Chest 2000;118 (5): 1355-64.

[22]. Silva Junior GT, Cardoso GP, Souza JBS, Alencar . RA, Monteiro EA, Villela C, et al. Prevalence of tuberculous pleuritis in unit of pleural diseases of the Antonio Pedro University Hospital, Pulmao RJ 2003;12(4):11-15.

[23]. Porcel JM, Esquerda A, Bielsa S. Diagnostic performance of adenosine deaminase activity in pleural fluid: A single-center experience with over 2100 consecutive patients.Eur. J. Inten. Med 2010;21(5):419-23.

[24]. Villegas MV, Labrada LA, Saravia NG. Evaluation of Polymerase Chain Reaction, Adenosine Deaminase, and Interferon-Gama in Pleural Fluid for the Differential Diagnosis of Pleural Tuberculosis. Chest 2000;118(5):1355-64.

[25]. De Oliveira HG, Rossatto ER, Prolla JC. Pleural fluid adenosine deaminase and lymphocyte proportion: clinical usefulness in the diagnosis of tuberculosis. Cytopathology1994;5(1): 27-32.

[26]. Antonangelo L, Vargas FS, Seiscento M, Bombarda S, Teixeira L, Sales RKB. Clinical and laboratory parameters in the differential diagnosis of pleural effusion secondary to tuberculosis or cancer. Clinics 2007;62(5): 585-590.

[27]. Zamalloa GA, Gomez T. Diagnostic accuracy of adenosine deaminase and lymphocyte proportion in pleural fluid for tuberculous pleurisy in different prevalence scenarios. PLos ONE 2012;7(6),1-8.

[28]. Gupta BK, Bharat V, Bandyopadhyay D. Role of Adenosine deaminase estimation in differentiation of tuberculous and nontuberculous exudative pleural effusions, J Clin Med Res 2010;2(2):79-84.

[29]. Gupta BK, Bharat V, Bandyopadhyay D. Sensitivity, specificity, negative and positive predictive values of Adenosine deaminase in patients of Tubercular and Non-tubercular Serosal Effusion in India, J.Clin Med Res 2010;2(3): 121-26.

[30]. Tuon FF, Higashino HR, Banks MI, Lopes F, Litvoc MN, Atomiya AN et al, Adenosine deaminase and tuberculous meningitis - A systematic review with meta-analysis, scand J Infec Dis 2010;42:198-207.

[31]. Gupta BK, Goel P, Baruah H, Shukla P, Kaur J. Cerebrospinal fluid Adenosine deaminase: its evaluation as a marker for diagnosing tuberculous meningitis in paediatric patients, IOSR-JDMS 2013;4(1):21-24 